\title{
Registration, Matching, and Data Fusion in 2D/3D Medical Imaging: Application to DSA and MRA
}

\author{
Maximilien Vermandel ${ }^{1}$, Nacim Betrouni ${ }^{2}$, Georges Palos ${ }^{2}$, Jean-Yves Gauvrit ${ }^{1}$, \\ Christian Vasseur ${ }^{2}$, and Jean Rousseau ${ }^{1}$ \\ ${ }^{1}$ Laboratoire de Biophysique - ITM, UPRES EA 1049, Pavillon Vancostenobel, University \\ Hospital, F-59037 cedex, Lille, France \\ \{m-vermandel, g-palos, jrousseau, jygauvrit\}@chru-lille.fr \\ ${ }^{2}$ Laboratoire I ${ }^{3} \mathrm{D}$, FRE-CNRS 2497, P2, USTL, 59655 cedex, Villeneuve d'Ascq, France \\ \{nb, Christian.Vasseur\}@i3d.univ-lille1.fr
}

\begin{abstract}
This paper deals with a new approach of registration in multimodal imaging. Modalities involved are Digital Subtracted Angiography (DSA, 2D) and Magnetic Resonance Angiography (MRA, 3D). Our approach is an hybrid one, mixing feature and intensity based approaches. This approach is based on the extraction of a anatomical referential common to both MRA and DSA. This step appears to be the "geometric-like" aspect. Then, a high level optimization scheme gives the best registration, using an iconic similarity measure. Several ways of matching planar and tomographic imaging are proposed through superimposition, point to point matching or 3D data fusion. The results obtained prove the methods efficiency in a clinical context.
\end{abstract}

\section{Introduction}

During the last decade, multimodality matching became more and more practicable thanks to the improvements in medical imaging and data processing techniques. As consequence, data fusion became a main center of interest in image assisted therapy. Currently, the complementarity of the different data available through all the existing imaging modalities brings more and more accuracy to anatomical and functional exploration. Thus, image matching is involved in treatment planning, in such a manner that "digital matching methods" [1] progressively replace the "mental" way of matching. Whatever the way of digitally matching method used, numerous preliminary steps are needed. Particularly, registration is the main step before matching or merging data. We can define registration as the establishment of a relationship between two datasets with a searching procedure. This relationship may be described through different kinds of transformation (rigid, affine, elastic). In this way, data matching and fusion are an exploitation of a such a transformation for localization (point to point matching) or visualization and analysis (data merging). Each method can be described using several criteria [2]. In this paper, we focus on data fusion and matching for images acquired through Magnetic Resonance Imaging (tomographic imaging) and X-Ray imaging (planar imaging), especially Magnetic Resonance Angiography (MRA) and Digital Subtracted Angiography (DSA). 2D/3D registration of MRA and DSA images find out a lot of medical applications. 
An example is the follow-up of treated aneurisms. Currently, after an aneurysm is diagnosed, an interventional procedure is carried out to place coils or glue within the aneurismal bag. Then, the treated aneurysm is followed-up during several years using MRA and/or DSA to observe and quantify a possible treatment failure. Most often, the follow-up is carried out by a mental matching, but an automatic or semi-automatic registration method would give a more objective and more accurate monitoring of the pathology. In the same neuroradiology context, MRA/DSA registration could be used to validate the new acquisition sequences in dynamic MRA, which would be an imaging alternative to X-ray examinations for the diagnosis of arteriovenous malformations (AVM). Currently, DSA remains the gold standard in vascular imaging. A final example is the radiosurgery of AVMs. The treatment planning is based on MRI or CT images and on DSA images, all these examination being performed with a stereotactic frame [3]. DSA/MRA registration could enable to avoid the stereotactic X-ray examination by using the first DSA examination obtained during the diagnosis step.

The Methods section deals with the registration approach. Accuracy and robustness of the registration will be evaluated and are presented through the Results section. Different matching solution will be then exposed.

\section{Method}

The problem of $2 \mathrm{D} / 3 \mathrm{D}$ registration is the optimization of the six-rigid body parameters describing the orientation of the 3D dataset. Many methods have been developed in order to solve the 2D/3D registration problem. Mainly, two classes of approaches can be distinguished: intensity based methods and feature based methods.

\subsection{Intensity-Based Approach}

The intensity based approaches [4], [5], [6], [7] are based on statistical dependence between the different modalities involved. Basically, intensity based approaches optimize the six-rigid parameters by generating digitally reconstructed radiographs (DRR) from the 3D dataset. These DDRs are compared to the planar images through a measure of similarity. Intensity based methods are well known for their high accuracy. Unfortunately, the DRRs generations dramatically increase the computing time. Furthermore, only CT images (not MRI) can be matched to the X-rays DSA.

\subsection{Feature-Based Approach}

The feature based approaches [8], [9], [10] are based on extraction of primitive figures from both modalities (as edges or skeleton). 2D and 3D primitives are registered by projecting the $3 \mathrm{D}$ figures and by minimizing the distance between the 2D and the projected 3D figures. Contrarily to the intensity based methods, feature approaches seem to be more robust and less time consuming. Nevertheless, the numerous preliminary steps needed (segmentation, reconstruction, skeleton extraction...) lead to a lower accuracy [11]). 


\subsection{Hybrid Approach}

Taking into account the clinical constraints in terms of accuracy and computing time, we have developed an hybrid approach, mixing intensity and feature based approaches. 3D reconstruction, as in the feature based method, is first performed, followed by the projection of the complete reconstructed structure (instead of the skeleton or isosurface) and the use of intensity based measure of similarity.

\section{Reconstruction}

The first step of the method is the segmentation of a vascular reference among all the MRA slices. A piece of the vascular tree is interactively selected on a Maximum Intensity Projection image (MIP). Then, the comparison of the segmented MIP image resulting from the selection with all the MRA slices enables us to extract the vascular structure (Fig. 1 and 2).
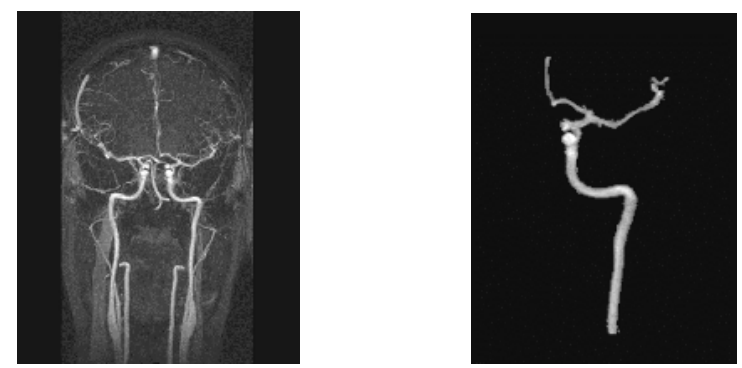

Fig. 1. A vessel is selected on a MIP image reconstructed from a MRA dataset.

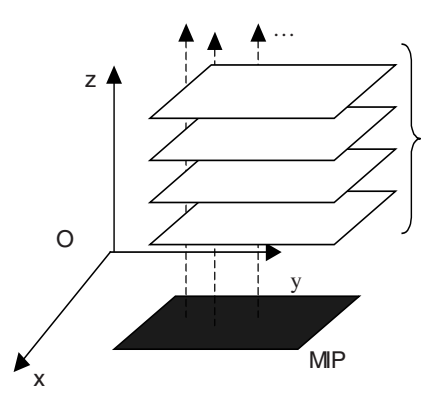

(a)

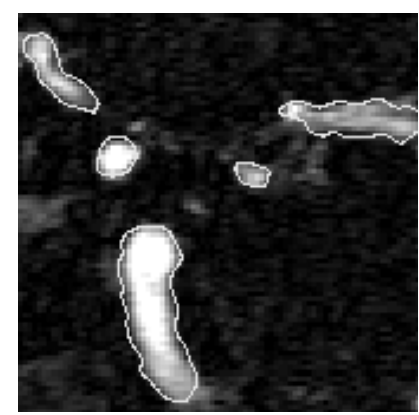

(b)

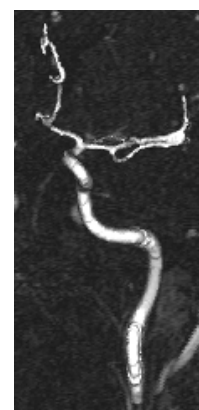

(c)

Fig. 2. The selected vessel is then compared to each slice (a) for being detected among all the dataset (b) and finally reconstructed (c).

Once the structure is obtained, a fuzzy set theory based method [12] is used to perform an accurate $3 \mathrm{D}$ reconstruction of the vessel. The approximately same piece of vessel is then selected on the DSA image. For selecting the vessel, region growing approach is applied on both MIP and DSA images. Thus, this anatomical object defines the common referential between both MRA and DSA modalities. 


\section{Similarity Measure and Optimization Scheme}

The optimal registration is obtained for the six rigid-body parameters giving the maximum similarity between the segmented DSA and the projection of the 3D reconstruction of the vascular MRA structure. The similarity measure used in the algorithm is the quadratic distance (1) between pixel gray level, where $P_{i, j}$ and $P^{\prime}{ }_{i, j}$ are the gray levels of the pixels placed in $(i, j)$ on image $I$ (e.g. DSA) and $I^{\prime}$ (e.g. projection).

$$
\text { Energy }=\sum_{i=1}^{I} \sum_{j=1}^{J}\left(P_{i, j}-P_{i, j}^{\prime^{2}}\right)^{2}
$$

Quadratic distance has been chosen because of its compatibility whatever the projection algorithm used, either binary or not. It must be noted that, in the current software implementation, projection of the 3D structure and the selected vessel on DSA are binary, so $P$ and $P^{\prime}$ can only take two values ( 0 or 1$)$. However, even if the data used for registration is binary, the energy is not necessary convex. To avoid possible local minima, we have used an optimization algorithm designed by Salazar and Toral [13]. The Hybrid Simulated Annealing method (HSA) is a Monte-Carlo like algorithm but leading to a computation time close to more usual methods (e.g. conjugate gradient). Once the maximum of similarity obtained, the position and the orientation of the common referential are known. Thus, transformation matrixes between the two modalities are available.

\section{Results}

To evaluate accuracy and robustness of the method, we have used the same experiments as in [11], where authors compare intensity-based and feature-based approaches, and in [7] where authors evaluate similarity measures. The phantom used for the experiments was designed with multimodality markers enabling measurements of the registration accuracy (Fig. 3).

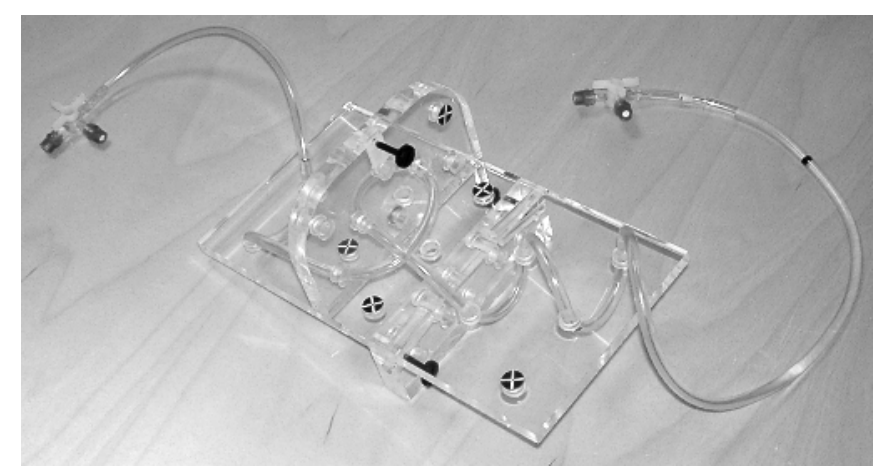

Fig. 3. Vascular phantom equipped with multimodality markers. 


\section{Robustness}

First of all, the optimal registration was computed. Then, starting positions for the registrations were chosen by perturbing the optimal position considered as gold standard value. Four experiments were performed with progressive increasing perturbation, as shown in table 1 . Note that in the experiment proposed by [11], there was no in-plane translation $(\delta X$ or $\delta Y$ ).

Table 1. Perturbation of the starting positions from the gold standard

\begin{tabular}{cllll}
\hline Experiment \# & \multicolumn{1}{c}{$\delta Z$} & $\delta 6$ & $\delta \varphi$ & $\delta \psi$ \\
\hline 1 & $+/-25 \mathrm{~mm}$ & $+/-4^{\circ}$ & $+/-4^{\circ}$ & $+/-4^{\circ}$ \\
2 & $+/-50 \mathrm{~mm}$ & $+/-8^{\circ}$ & $+/-8^{\circ}$ & $+/-8^{\circ}$ \\
3 & $+/-75 \mathrm{~mm}$ & $+/-12^{\circ}$ & $+/-12^{\circ}$ & $+/-12^{\circ}$ \\
4 & $+/-100 \mathrm{~mm}$ & $+/-16^{\circ}$ & $+/-16^{\circ}$ & $+/-16^{\circ}$ \\
\hline
\end{tabular}

Using the rotation and translation matrix resulting for each registration, we have computed the 3D coordinates of the markers. Then, the re-projection distance [14] (Fig. 4) for each marker was computed and the average RMS error was obtained for a given registration.

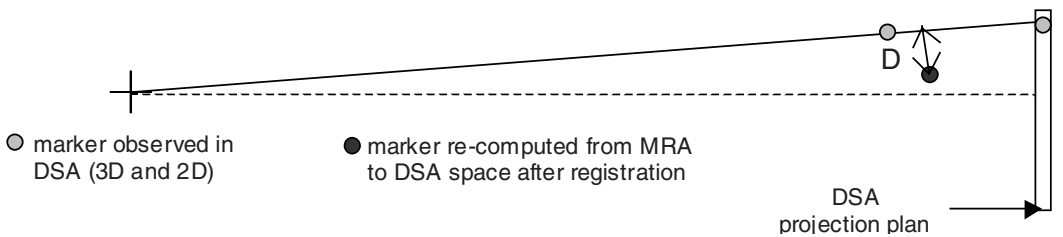

Fig. 4. Computation of the re-projection distance.

If the average RMS error for a particular registration was less than $4 \mathrm{~mm}$, the registration was judged as a successful registration. Therefore, the number of successful registration compared to the number of all the registrations performed for an experiment level gave an evaluation of the robustness.

\section{Accuracy}

For the accuracy, only successful registrations were kept to compute an average RMS error for a given experiment level. Results for robustness and accuracy are shown on Fig. 5.

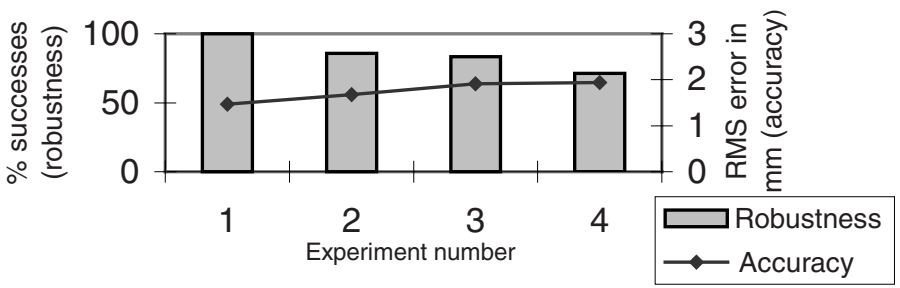

Fig. 5. Plot of the accuracy and reliability of the method.

The satisfactory results show that this approach combines high robustness (even in the worst condition of initialization) and high accuracy, close to the accuracy found by [11] for intensity based method. 


\section{Matching Solutions}

Once the registration is achieved, different solutions have been developed to match MRA and DSA data, which are 2D-2D, 2D/2D-3D and 3D-3D.

\subsection{D to 2D Data Matching}

From the six rigid body parameters deduced from the registration, we can virtually transpose the MRA dataset to perform a "virtual DSA" using a simple MIP algorithm with a conic projection (instead of parallel projection). Then, original DSA (with or without cine mode) and virtual DSA can be superimposed using transparency display (Fig. 6).

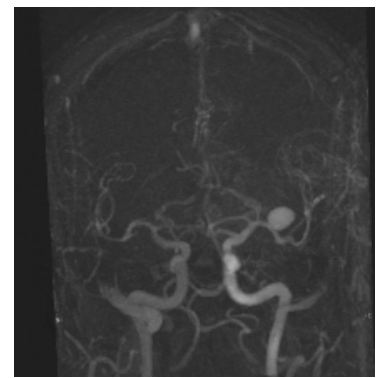

(a)

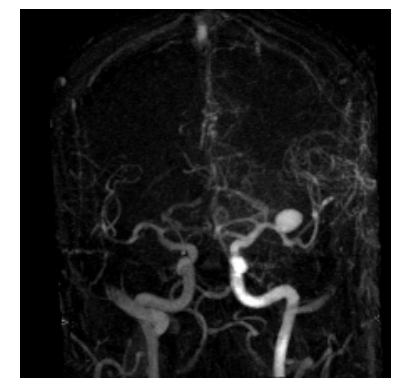

(b)

Fig. 6. Superimposition of virtual and original DSA, (a) virtual (b) virtual and original.

\subsection{D/2D to 3D Data Matching: Real Time Navigation}

A standard DSA examination is made of at least 2 incidences which are registered with the 3D MRA reconstruction. Therefore, we can use these two incidences as a stereoscopic process, and we can navigate between DSA and MRA, using epipolarity constraints [15] (Fig 7).
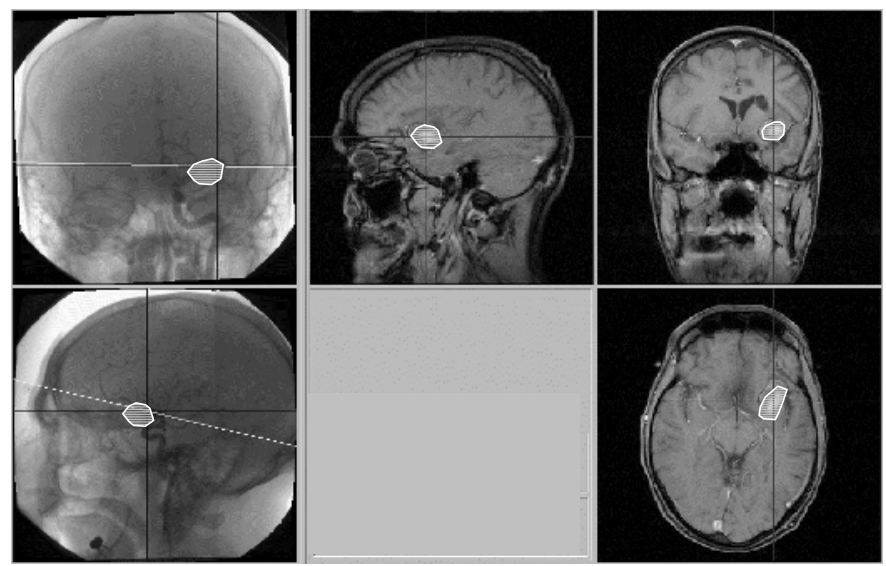

Fig. 7. DSA to MRI/MRA navigation and contouring. 


\subsection{D to 3D Matching}

When a sufficient number of DSA incidences is available and after the registration of each view within the common referential, we can perform a 3D reconstruction of the DSA data. Two ways of reconstruction are possible : tomographic (which needs a large number of views) and a feature based reconstruction technique (which needs at least 3 different views [16]. Therefore, DSA reconstruction can be transposed on the corresponding MRA slices (Fig. 8).
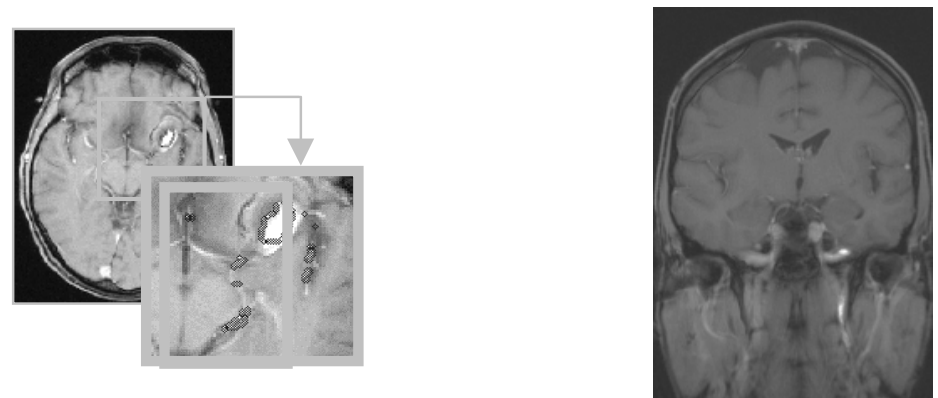

Fig. 8. Merging DSA data to MRI slices from feature based reconstruction and from tomographic reconstruction.

\section{Discussion - Conclusion}

Considering the robustness and accuracy obtained, the method exposed seems to be very efficient for $2 \mathrm{D}-3 \mathrm{D}$ registration. Compared to those obtained by McLaughlin for the other approaches, results tend to claim that a new intermediate solution to 2D/3D registration has been developed. In one hand, the feature-based methods are very sensitive to the quality of both $2 \mathrm{D}$ and $3 \mathrm{D}$ skeletonisation algorithm but offers real high results in term of computation time. On the other hand, because of its accuracy, intensity-based methods are very computationally intensive. Thus, they can not be used for real time clinical application. The approach offers an accuracy similar to the intensity-based method while being far less computationally intensive.

An another breakthrough of the method is its ability to realize an accurate registration with only one DSA incidence. It makes it possible to acquire images without any geometrical constraint (table height, source to intensifier distance, field of view...) at each acquisition and without external localization frame. This property makes the approach perfectly suitable for applications in any clinical context (diagnosis or therapy).

Hybrid approach is currently being clinically evaluated to validate the accuracy of the method in real clinical conditions and to analyze the medical breakthrough brought by the approach for different studies (aneurysm follow-up, arterioveinous malformation diagnosis, ...). If it appears to be necessary, the next implementation will use a gray level projection algorithm instead of the binary one, and an ICP (Iterative Closest Point) [10] initialization will be performed before the optimization process. 


\section{References}

1. Brown L.G.: A survey of image registration techniques. ACM Computing Surveys, vol. 24, nº (1992) 325-376

2. Maintz J.B.A, Viergever M.A.: A survey of medical image registration. Medical Image Analysis, vol. 2, n (1998) 1-36

3. Gibon D., Coste E., Vial S., Vasseur C., Rousseau J.: Stereotactic localization in medical imaging. A technical and methodological review. J. Rad. Surger. vol. 2, n³ (1999)167180

4. Penney G.P., Weese J., Little J.A., Desmedt P., Hill D.L., Hawkes D.J.: A comparison of similarity measures for use in 2D-3D medical image registration. IEEE TMI vol. 17, ${ }^{\circ} 4$ (1998) 586-595

5. Penney G.P.: Registration of tomographic images to X-Ray projections for use in image guided interventions, $\mathrm{PhD}$ thesis of the University College of London, (2000).

6. Weese J., Buzug T.M., Lorenz C., Fassnacht C.: An approach to 2D/3D registration of a vertebra in 2D X-Ray fluoroscopies with 3D CT images. CVRMed/MRCAS'97, LNCS, Vol. 1205, Springer (1997) 119-128

7. Hipwell J.H., Penney G.P., Cox T.C., Byrne J.V., Hawkes D.J.: 2D-3D Intensity Based Registration of DSA and MRA - A Comparison of Similarity Measures, MICCAI 2002, LNCS, Vol. 2489, Springer (2002) 501-508

8. Hamadeh A., Cinquin P.: Kinematic study of lumbar sine using functional radiographies and 3D/2D registration. CVRMed/MRCAS'97, LNCS, Vol. 1205, Springer, (1997) 109118

9. Feldmar J., Malandain G., Ayache N., Fernàndez-Vidal S., Maurincomme E., Trousset Y.: Matching 3D MR Angiography data and 2D X-Ray Angiograms. CVRMed/MRCAS'97, LNCS, Vol. 1205, Springer (1997) 129-138

10. Betting F., Feldmar J.: 3D/2D projective registration of anatomical surfaces with their projections. XIV International Conference on Information Processing in Medical Imaging (1995) 275-286

11. Mc Laughlin R.A., Hipwell J., Penney G.P., Rhode K., Chung A., Noble J.A., Hawkes D.J.: Intensity-Based Registration Versus Feature-Based Registration for Neurointerventions. Medical Image Understanding and Analysis, Lecture notes in Proceedings of MIUA 2001 (2001)

12. Vial S., Gibon D., Vasseur C., Rousseau J.: Volume delineation by fusion of fuzzy set obtained from multiplanar tomographic images. IEEE TMI, vol. 20, n 12 (2001) 13621372

13. Salazar R., Toral R.: Simulated Annealing using Hybrid Monte Carlo. Journal of Statistical Physics, vol. 89 (1997)

14. Masutani Y., Dohi T., Yamane F., Iseki H., Takakura K.: Interactive virtualised display system for intravascular neurosurgery, CVRMed/MRCAS'97, LNCS, Vol. 1205, Springer (1997) 428-435

15. Vermandel M., Kulik C., Leclerc X., Rousseau J., Vasseur C.: Matching of projection imaging and tomographic imaging, Lect. Note. in Proc. SPIE Medical Imaging, vol. 4681 (2002) 55-64

16. Coste E., Vasseur C., Rousseau J.: 3D reconstruction of the cerebral arterial network. Medical Physics (1999) Vol. 26, n9 1783-1793 